\title{
On the connections among Fibonacci, Pell, Jacobsthal and Padovan numbers
}

\author{
Ömür Deveci \\ Department of Mathematics, Faculty of Science and Letters, \\ Kafkas University, 36100, Turkey \\ e-mail: odeveci36@hotmail.com
}

Received: 18 March 2021

Revised: 23 May 2021

Accepted: 7 June 2021

\begin{abstract}
In this paper, we define the Fibonacci-Jacobsthal, Padovan-Fibonacci, Pell-Fibonacci, Pell-Jacobsthal, Padovan-Pell and Padovan-Jacobsthal sequences which are directly related with the Fibonacci, Jacobsthal, Pell and Padovan numbers and give their structural properties by matrix methods. Then we obtain new relationships between Fibonacci, Jacobsthal, Pell and Padovan numbers.
\end{abstract}

Keywords: Fibonacci sequence, Jacobsthal sequence, Pell sequence, Padovan sequence, Matrix, Representation.

2020 Mathematics Subject Classification: 11K31, 11C20, 15A15.

\section{Introduction}

The well-known Fibonacci, Jacobsthal, Pell and Padovan sequences are defined by the following recurrence relations, respectively:

$$
\begin{aligned}
& F_{n}=F_{n-1}+F_{n-2} \text { for } n \geq 2 \text { in which } F_{0}=0 \text { and } F_{1}=1, \\
& J_{n}=J_{n-1}+2 J_{n-2} \text { for } n \geq 2 \text { in which } J_{0}=0 \text { and } J_{1}=1, \\
& P_{n}=2 P_{n-1}+P_{n-2} \text { for } n \geq 2 \text { in which } P_{0}=0 \text { and } P_{1}=1,
\end{aligned}
$$

and

$$
P a_{n}=P a_{n-2}+P a_{n-3} \text { for } n \geq 3 \text { in which } P a_{0}=P a_{1}=P a_{2}=1 .
$$

It is easy to see that the characteristic polynomials of the Fibonacci, Jacobsthal, Pell and Padovan sequences are $f_{1}(x)=x^{2}-x-1, f_{2}(x)=x^{2}-x-2, f_{3}(x)=x^{2}-2 x-1$ and $f_{4}(x)=x^{3}-x-1$, respectively. We use these in the next section. 
Let the $(n+k)$-th term of a sequence be defined recursively by a linear combination of the preceding $k$ terms:

$$
a_{n+k}=c_{0} a_{n}+c_{1} a_{n+1}+\cdots+c_{k-1} a_{n+k-1},
$$

where $c_{0}, c_{1}, \ldots, c_{k-1}$ are real constants. In [13], Kalman derived a number of closed-form formulas for the generalized sequence by the companion matrix method as follows:

Let $A$ be a matrix of order $k$ as follows:

$$
A=\left[a_{i, j}\right]_{k \times k}=\left[\begin{array}{cccccc}
0 & 1 & 0 & \cdots & 0 & 0 \\
0 & 0 & 1 & \cdots & 0 & 0 \\
0 & 0 & 0 & \cdots & 0 & 0 \\
\vdots & \vdots & \vdots & \ddots & \vdots & \vdots \\
0 & 0 & 0 & \cdots & 0 & 1 \\
c_{0} & c_{1} & c_{2} & \cdots & c_{k-2} & c_{k-1}
\end{array}\right],
$$

then

$$
A^{n}\left[\begin{array}{c}
a_{0} \\
a_{1} \\
\vdots \\
a_{k-1}
\end{array}\right]=\left[\begin{array}{c}
a_{n} \\
a_{n+1} \\
\vdots \\
a_{n+k-1}
\end{array}\right]
$$

for $n \geqslant 0$.

Number theoretic properties such as these obtained from homogeneous linear recurrence relations relevant to this paper have been studied recently by many authors: see, for example, $[1,4$, 8-12,20-22,24]. In [5-7,14-17,23,25], the authors defined some linear recurrence sequences and gave their various properties by matrix methods. In this paper, we discuss connections between the Fibonacci, Jacobsthal, Pell, and Padovan numbers.

Firstly, we define the Fibonacci-Jacobsthal, Padovan-Fibonacci, Pell-Fibonacci, PellJacobsthal, Padovan-Pell, and Padovan-Jacobsthal sequences and then we give recurrence relations among these sequences and the Fibonacci, Jacobsthal, Pell, and Padovan sequences. Also, we give the relations between the generating matrices of sequences defined and the elements of Fibonacci, Jacobsthal, Pell, and Padovan sequences.

Furthermore, using the generating matrices and the generating functions of sequences defined, we obtain their structural properties such as the Binet formulas, the exponential and combinatorial representations which are intimately connected with the Fibonacci, Jacobsthal, Pell, and Padovan numbers. Finally, we derive the permanental, determinantal representations and the sums of the Fibonacci-Jacobsthal, Padovan-Fibonacci, Pell-Fibonacci, Pell-Jacobsthal, Padovan-Pell, and Padovan-Jacobsthal numbers by the certain matrices.

\section{Main results}

Define the Fibonacci-Jacobsthal, Padovan-Fibonacci, Pell-Fibonacci, Pell-Jacobsthal, PadovanPell, and Padovan-Jacobsthal sequences as follows, respectively: 


$$
F-J(n+4)=2 F-J(n+3)+2 F-J(n+2)-3 F-J(n+1)-2 F-J(n)
$$

for $n \geq 0$ in which $F-J(0)=F-J(1)=F-J(2)=0$ and $F-J(3)=1$,

$$
P a-F(n+5)=P a-F(n+4)+2 P a-F(n+3)-2 P a-F(n+1)-P a-F(n)
$$

for $n \geq 0$ in which $P a-F(0)=P a-F(1)=P a-F(2)=P a-F(3)=0$ and $P a-F(4)=1$,

$$
P-F(n+4)=3 P-F(n+3)-3 P-F(n+1)-P-F(n)
$$

for $n \geq 0$ in which $P-F(0)=P-F(1)=P-F(2)=0$ and $P-F(3)=1$,

$$
P-J(n+4)=3 P-J(n+3)+P-J(n+2)-5 P-J(n+1)-2 P-J(n)
$$

for $n \geq 0$ in which $P-J(0)=P-J(1)=P-J(2)=0$ and $P-J(3)=1$,

$$
P a-P(n+5)=2 P a-P(n+4)+2 P a-P(n+3)-P a-P(n+2)-3 P a-P(n+1)-P a-P(n),
$$

for $n \geq 0$ in which $P a-P(0)=P a-P(1)=P a-P(2)=P a-P(3)=0$ and $P a-P(4)=1$, and

$$
P a-J(n+5)=P a-J(n+4)+3 P a-J(n+3)-3 P a-J(n+1)-2 P a-J(n),
$$

for $n \geq 0$ in which $P a-J(0)=P a-J(1)=P a-J(2)=P a-J(3)=0$ and $P a-J(4)=1$.

First we consider relationships between the above sequences and the Fibonacci, Jacobsthal, Pell, and Padovan sequences.

Theorem 2.1. Let $F-J(n), P a-F(n), P-F(n), P-J(n), P a-P(n)$ and $P a-J(n)$ be the nth Fibonacci-Jacobsthal, Padovan-Fibonacci, Pell-Fibonacci, Pell-Jacobsthal, Padovan-Pell, and Padovan-Jacobsthal numbers, respectively, then

$$
\begin{aligned}
F-J(n) & =J_{n}-F_{n} \quad \text { for } n \geq 0, \\
P a-F(n+2) & =F_{n+1}-P a_{n} \quad \text { for } n \geq 0, \\
P-F(n+1) & =P_{n}-F_{n} \quad \text { for } n \geq 0, \\
P-J(n) & =\sum_{i=0}^{n-1}\left(P_{i}-J_{i}\right) \quad \text { for } n \geq 1, \\
4 P a-P(n+5)+P a-P(n+4) & =P_{n+4}-P a_{n+3}-P a_{n} \quad \text { for } n \geq 0, \quad \text { and } \\
2 P a-J(n+2)+P a-J(n+1) & =J_{n+1}-P a_{n} \quad \text { for } n \geq 0 .
\end{aligned}
$$

Proof. Let us consider the first equation. We will use the induction method on $n$. It is clear that $F-J(0)=J_{0}-F_{0}=0$. Now we assume that the equation holds for $n \geq 0$. Then we show that the equation holds for $n+1$. Since the characteristic polynomial of the Fibonacci-Jacobsthal sequence is $p(x)=x^{4}-2 x^{3}-2 x^{2}+3 x+2$ and $p(x)=f_{1}(x) f_{2}(x)$ where $f_{1}(x)$ and $f_{2}(x)$ are characteristic polynomials of the Fibonacci and Jacobsthal sequence, respectively, we obtain the following relations:

$$
\begin{aligned}
& F_{n+4}=2 F_{n+3}+2 F_{n+2}-3 F_{n+1}-2 F_{n}, \\
& J_{n+4}=2 J_{n+3}+2 J_{n+2}-3 J_{n+1}-2 J_{n} .
\end{aligned}
$$

for $n \geq 0$. Thus, by a simple calculation, we have the conclusion.

The proofs of other equations are similar to the above and are omitted. 


\subsection{Companion matrices}

By the recurrence relations (1)-(6), we can write the following companion matrices, respectively:

$$
\begin{aligned}
M_{1}= & {\left[\begin{array}{llll}
2 & 2 & -3 & -2 \\
1 & 0 & 0 & 0 \\
0 & 1 & 0 & 0 \\
0 & 0 & 1 & 0
\end{array}\right], } \\
M_{2}= & {\left[\begin{array}{lllll}
1 & 2 & 0 & -2 & -1 \\
1 & 0 & 0 & 0 & 0 \\
0 & 1 & 0 & 0 & 0 \\
0 & 0 & 1 & 0 & 0 \\
0 & 0 & 0 & 1 & 0
\end{array}\right], } \\
M_{3}= & {\left[\begin{array}{llll}
3 & 0 & -3 & -1 \\
1 & 0 & 0 & 0 \\
0 & 1 & 0 & 0 \\
0 & 0 & 1 & 0
\end{array}\right] } \\
M_{4}= & {\left[\begin{array}{lllll}
3 & 1 & -5 & -2 \\
1 & 0 & 0 & 0 \\
0 & 1 & 0 & 0 \\
0 & 0 & 1 & 0
\end{array}\right] } \\
M_{5}= & {\left[\begin{array}{lllll}
2 & 2 & -1 & -3 \\
1 & 0 & 0 & 0 & 0 \\
0 & 1 & 0 & 0 & 0 \\
0 & 0 & 1 & 0 & 0 \\
0 & 0 & 0 & 1 & 0
\end{array}\right], }
\end{aligned}
$$

and

$$
M_{6}=\left[\begin{array}{lllll}
1 & 3 & 0 & -3 & -2 \\
1 & 0 & 0 & 0 & 0 \\
0 & 1 & 0 & 0 & 0 \\
0 & 0 & 1 & 0 & 0 \\
0 & 0 & 0 & 1 & 0
\end{array}\right]
$$

The matrices $M_{1}, M_{2}, M_{3}, M_{4}, M_{5}$ and $M_{6}$ are said to be the Fibonacci-Jacobsthal, PadovanFibonacci, Pell-Fibonacci, Pell-Jacobsthal, Padovan-Pell, and Padovan-Jacobsthal, respectively. Let $F-J(n), P a-F(n), P-F(n), P-J(n), P a-P(n)$ and $P a-J(n)$ be denoted by $x_{n}^{1}$, $x_{n}^{2}, x_{n}^{3}, x_{n}^{4}, x_{n}^{5}$ and $x_{n}^{6}$, respectively. Since $F_{0}, J_{0}, P_{0}$ and $P a_{0}$ are initial values of the sequences $\left\{F_{n}\right\},\left\{J_{n}\right\},\left\{P_{n}\right\}$ and $\left\{P a_{n}\right\}$, respectively, we consider the multiplicative order of the generating matrices for suitable values of $n$. By mathematical induction on $n$, we derive: 


$$
\begin{aligned}
& \left(M_{1}\right)^{n}=\left[\begin{array}{cccc}
x_{n+3}^{1} & F_{n+1}+(-1)^{n+1} & F_{n+3}-x_{n+4}^{1} & F_{n}-x_{n+3}^{1}+(-1)^{n} \\
x_{n+2}^{1} & F_{n}+(-1)^{n} & F_{n+2}-x_{n+3}^{1} & F_{n-1}-x_{n+2}^{1}+(-1)^{n+2} \\
x_{n+1}^{1} & F_{n-1}+(-1)^{n+1} & F_{n+1}-x_{n+2}^{1} & F_{n-2}-x_{n+1}^{1}+(-1)^{n} \\
x_{n}^{1} & F_{n-2}+(-1)^{n} & F_{n}-x_{n+1}^{1} & F_{n-3}-x_{n}^{1}+(-1)^{n+1}
\end{array}\right] \text { for } n \geq 3, \\
& \left(M_{2}\right)^{n}=\left[\begin{array}{ccccc}
x_{n+4}^{2} & P a_{n-1}+x_{n+3}^{2} & P a_{n}-x_{n+4}^{2} & P a_{n+1}-x_{n+5}^{2} & -x_{n+3}^{2} \\
x_{n+3}^{2} & P a_{n-2}+x_{n+2}^{2} & P a_{n-1}-x_{n+3}^{2} & P a_{n}-x_{n+4}^{2} & -x_{n+2}^{2} \\
x_{n+2}^{2} & P a_{n-3}+x_{n+1}^{2} & P a_{n-2}-x_{n+2}^{2} & P a_{n-1}-x_{n+3}^{2} & -x_{n+1}^{2} \\
x_{n+1}^{2} & P a_{n-4}+x_{n}^{2} & P a_{n-3}-x_{n+1}^{2} & P a_{n-2}-x_{n+2}^{2} & -x_{n}^{2} \\
x_{n}^{2} & P a_{n-5}+x_{n-1}^{2} & P a_{n-4}-x_{n}^{2} & P a_{n-3}-x_{n+1}^{2} & -x_{n-1}^{2}
\end{array}\right] \text { for } n \geq 5, \\
& \left(M_{3}\right)^{n}=\left[\begin{array}{cccc}
x_{n+3}^{3} & F_{n+2}+x_{n+2}^{3}-x_{n+3}^{3} & F_{n+3}+x_{n+3}^{3}-x_{n+4}^{3} & -x_{n+2}^{3} \\
x_{n+2}^{3} & F_{n+1}+x_{n+1}^{3}-x_{n+2}^{3} & F_{n+2}+x_{n+2}^{3}-x_{n+3}^{3} & -x_{n+1}^{3} \\
x_{n+1}^{3} & F_{n}+x_{n}^{3}-x_{n+1}^{3} & F_{n+1}+x_{n+1}^{3}-x_{n+2}^{3} & -x_{n}^{3} \\
x_{n}^{3} & F_{n-1}+x_{n-1}^{3}-x_{n}^{3} & F_{n}+x_{n}^{3}-x_{n+1}^{3} & -x_{n-1}^{3}
\end{array}\right] \text { for } n \geq 1, \\
& \left(M_{4}\right)^{n}=\left[\begin{array}{cccc}
x_{n+3}^{4} & J_{n+2}+x_{n+2}^{4}-x_{n+3}^{4} & J_{n+3}-x_{n+4}^{4} & -2 x_{n+2}^{4} \\
x_{n+2}^{4} & J_{n+1}+x_{n+1}^{4}-x_{n+2}^{4} & J_{n+2}-x_{n+3}^{4} & -2 x_{n+1}^{4} \\
x_{n+1}^{4} & J_{n}+x_{n}^{4}-x_{n+1}^{4} & J_{n+1}-x_{n+2}^{4} & -2 x_{n}^{4} \\
x_{n}^{4} & J_{n-1}+x_{n-1}^{4}-x_{n}^{4} & J_{n}-x_{n+1}^{4} & -2 x_{n-1}^{4}
\end{array}\right] \text { for } n \geq 1, \\
& \left(M_{5}\right)^{n}=\left[\begin{array}{ccccc}
x_{n+4}^{5} & P a_{n-1}+x_{n+3}^{5} & P a_{n}-x_{n+4}^{5} & P a_{n-2}-x_{n+4}^{5}-x_{n+3}^{5} & -x_{n+3}^{5} \\
x_{n+3}^{5} & P a_{n-2}+x_{n+2}^{5} & P a_{n-1}-x_{n+3}^{5} & P a_{n-3}-x_{n+3}^{5}-x_{n+2}^{5} & -x_{n+2}^{5} \\
x_{n+2}^{5} & P a_{n-3}+x_{n+1}^{5} & P a_{n-2}-x_{n+2}^{5} & P a_{n-4}-x_{n+2}^{5}-x_{n+1}^{5} & -x_{n+1}^{5} \\
x_{n+1}^{5} & P a_{n-4}+x_{n}^{5} & P a_{n-3}-x_{n+1}^{5} & P a_{n-5}-x_{n+1}^{5}-x_{n}^{5} & -x_{n}^{5} \\
x_{n}^{5} & P a_{n-5}+x_{n-1}^{5} & P a_{n-4}-x_{n}^{5} & P a_{n-6}-x_{n}^{5}-x_{n-1}^{5} & -x_{n-1}^{5}
\end{array}\right] \quad \text { for } n \geq 6
\end{aligned}
$$

and

$$
\left(M_{6}\right)^{n}=\left[\begin{array}{ccccc}
x_{n+4}^{6} & x_{n+5}^{6}-x_{n+4}^{6} & P a_{n}-x_{n+4}^{6} & P a_{n+1}-x_{n+5}^{6} & -2 x_{n+3}^{6} \\
x_{n+3}^{6} & x_{n+4}^{6}-x_{n+3}^{6} & P a_{n-1}-x_{n+3}^{6} & P a_{n}-x_{n+4}^{6} & -2 x_{n+2}^{6} \\
x_{n+2}^{6} & x_{n+3}^{6}-x_{n+2}^{6} & P a_{n-2}-x_{n+2}^{6} & P a_{n-1}-x_{n+3}^{6} & -2 x_{n+1}^{6} \\
x_{n+1}^{6} & x_{n+2}^{6}-x_{n+1}^{6} & P a_{n-3}-x_{n+1}^{6} & P a_{n-2}-x_{n+2}^{6} & -2 x_{n}^{6} \\
x_{n}^{6} & x_{n+1}^{6}-x_{n}^{6} & P a_{n-4}-x_{n}^{6} & P a_{n-3}-x_{n+1}^{6} & -2 x_{n-1}^{6}
\end{array}\right] \text { for } n \geq 4 .
$$

\subsection{Binet formulas}

Now we concentrate on finding the Binet formulas for the Fibonacci-Jacobsthal, PadovanFibonacci, Pell-Fibonacci, Pell-Jacobsthal, Padovan-Pell, and Padovan-Jacobsthal numbers. It is clear that each of the eigenvalues of the matrices $M_{1}, M_{2}, M_{3}, M_{4}, M_{5}$ and $M_{6}$ are distinct, respectively. Let $\left\{\lambda_{1}^{(1)}, \lambda_{2}^{(1)}, \lambda_{3}^{(1)}, \lambda_{4}^{(1)}\right\},\left\{\lambda_{1}^{(2)}, \lambda_{2}^{(2)}, \lambda_{3}^{(2)}, \lambda_{4}^{(2)}, \lambda_{5}^{(2)}\right\},\left\{\lambda_{1}^{(3)}, \lambda_{2}^{(3)}, \lambda_{3}^{(3)}, \lambda_{4}^{(3)}\right\}$, 
$\left\{\lambda_{1}^{(4)}, \lambda_{2}^{(4)}, \lambda_{3}^{(4)}, \lambda_{4}^{(4)}\right\},\left\{\lambda_{1}^{(5)}, \lambda_{2}^{(5)}, \lambda_{3}^{(5)}, \lambda_{4}^{(5)}, \lambda_{5}^{(5)}\right\}$ and $\left\{\lambda_{1}^{(6)}, \lambda_{2}^{(6)}, \lambda_{3}^{(6)}, \lambda_{4}^{(6)}, \lambda_{5}^{(6)}\right\}$ be the sets of the eigenvalues of the matrices $M_{1}, M_{2}, M_{3}, M_{4}, M_{5}$ and $M_{6}$, respectively, and let $V_{k}^{(u)}$ be a $k \times k$ Vandermonde matrix (where $k=4$ for $u=1,3,4$; and $k=5$ for $u=2,5,6$ ) as follows:

$$
V_{k}^{(u)}=\left[\begin{array}{cccc}
\left(\lambda_{1}^{(u)}\right)^{k-1} & \left(\lambda_{2}^{(u)}\right)^{k-1} & \cdots & \left(\lambda_{k}^{(u)}\right)^{k-1} \\
\left(\lambda_{1}^{(u)}\right)^{k-2} & \left(\lambda_{2}^{(u)}\right)^{k-2} & \cdots & \left(\lambda_{k}^{(u)}\right)^{k-2} \\
\vdots & \vdots & \ddots & \vdots \\
1 & 1 & \cdots & 1
\end{array}\right] .
$$

Assume that

$$
W_{k}^{(u, i)}=\left[\begin{array}{c}
\left(\lambda_{1}^{(u)}\right)^{n+k-i} \\
\left(\lambda_{2}^{(u)}\right)^{n+k-i} \\
\vdots \\
\left(\lambda_{k}^{(u)}\right)^{n+k-i}
\end{array}\right]
$$

and $V_{k}^{(u, i, j)}$ is a $k \times k$ matrix obtained from $V_{k}^{(u)}$ by replacing the $j$-th column of $V_{k}^{(u)}$ by $W_{k}^{(u, i)}$.

Theorem 2.2. Let $\left(M_{u}\right)^{n}=m_{i, j}^{(u, n)}$, then

$$
m_{i, j}^{(u, n)}=\frac{\operatorname{det} V_{k}^{(u, i, j)}}{\operatorname{det} V_{k}^{(u)}},
$$

where $k=4$ for $u=1$ and $n \geq 3 ; k=4$ for $u=2$ and $n \geq 5 ; k=4$ for $u=3,4$ and $n \geq 1$; $k=5$ for $u=5$ and $n \geq 6$; and $k=5$ for $u=6$ and $n \geq 4$.

Proof. Let us consider the matrix $M_{2}$. Since the eigenvalues of the matrix $M_{2}$ are distinct, $M_{2}$ is diagonalizable. Let $D_{2}=\operatorname{diag}\left(\lambda_{1}^{(2)}, \lambda_{2}^{(2)}, \lambda_{3}^{(2)}, \lambda_{4}^{(2)}, \lambda_{5}^{(2)}\right)$, then it is readily seen that $M_{2} V_{5}^{(2)}=V_{5}^{(2)} D_{2}$. Since the matrix $V_{5}^{(2)}$ is invertible, $\left(V_{5}^{(2)}\right)^{-1} M_{2} V_{5}^{(2)}=D_{2}$. Therefore, $M_{2}$ is similar to $D_{2}$; hence, $\left(M_{2}\right)^{n}\left(V_{5}^{(2)}\right)^{n}=\left(V_{5}^{(2)}\right)^{n}\left(D_{2}\right)^{n}$ for $n \geq 5$. So we have the following linear system of equations:

$$
\left\{\begin{array}{l}
m_{i, 1}^{(2, n)}\left(\lambda_{1}^{(2)}\right)^{4}+m_{i, 2}^{(2, n)}\left(\lambda_{1}^{(2)}\right)^{3}+m_{i, 3}^{(2, n)}\left(\lambda_{1}^{(2)}\right)^{2}+m_{i, 4}^{(2, n)}\left(\lambda_{1}^{(2)}\right)+m_{i, 5}^{(2, n)}=\left(\lambda_{1}^{(2)}\right)^{n+5-i} \\
m_{i, 1}^{(2, n)}\left(\lambda_{2}^{(2)}\right)^{4}+m_{i, 2}^{(2, n)}\left(\lambda_{2}^{(2)}\right)^{3}+m_{i, 3}^{(2, n)}\left(\lambda_{2}^{(2)}\right)^{2}+m_{i, 4}^{(2, n)}\left(\lambda_{2}^{(2)}\right)+m_{i, 5}^{(2, n)}=\left(\lambda_{2}^{(2)}\right)^{n+5-i} \\
m_{i, 1}^{(2, n)}\left(\lambda_{3}^{(2)}\right)^{4}+m_{i, 2}^{(2, n)}\left(\lambda_{3}^{(2)}\right)^{3}+m_{i, 3}^{(2, n)}\left(\lambda_{3}^{(2)}\right)^{2}+m_{i, 4}^{(2, n)}\left(\lambda_{3}^{(2)}\right)+m_{i, 5}^{(2, n)}=\left(\lambda_{3}^{(2)}\right)^{n+5-i} . \\
m_{i, 1}^{(2, n)}\left(\lambda_{4}^{(2)}\right)^{4}+m_{i, 2}^{(2, n)}\left(\lambda_{4}^{(2)}\right)^{3}+m_{i, 3}^{(2, n)}\left(\lambda_{4}^{(2)}\right)^{2}+m_{i, 4}^{(2, n)}\left(\lambda_{4}^{(2)}\right)+m_{i, 5}^{(2, n)}=\left(\lambda_{4}^{(2)}\right)^{n+5-i} \\
m_{i, 1}^{(2, n)}\left(\lambda_{5}^{(2)}\right)^{4}+m_{i, 2}^{(2, n)}\left(\lambda_{5}^{(2)}\right)^{3}+m_{i, 3}^{(2, n)}\left(\lambda_{5}^{(2)}\right)^{2}+m_{i, 4}^{(2, n)}\left(\lambda_{5}^{(2)}\right)+m_{i, 5}^{(2, n)}=\left(\lambda_{5}^{(2)}\right)^{n+5-i}
\end{array} .\right.
$$

Then, for $i, j=1,2,3,4,5$, we obtain

$$
m_{i, j}^{(2, n)}=\frac{\operatorname{det} V_{5}^{(2, i, j)}}{\operatorname{det} V_{5}^{(2)}} .
$$

The proofs for other matrices are similar to the above and are omitted. 
Corollary 2.1. Let $F-J(n), P a-F(n), P-F(n), P-J(n), P a-P(n)$ and $P a-J(n)$ be the nth Fibonacci-Jacobsthal, Padovan-Fibonacci, Pell-Fibonacci, Pell-Jacobsthal, Padovan-Pell, and Padovan-Jacobsthal numbers, respectively, then:

$$
\begin{gathered}
F-J(n)=\frac{\operatorname{det} V_{4}^{(1,4,1)}}{\operatorname{det} V_{4}^{(1)}} \text { for } n \geq 3, \\
P a-F(n)=\frac{\operatorname{det} V_{5}^{(2,5,1)}}{\operatorname{det} V_{5}^{(2)}}=-\frac{\operatorname{det} V_{5}^{(2,4,5)}}{\operatorname{det} V_{5}^{(2)}} \quad \text { for } n \geq 5, \\
P-F(n)=\frac{\operatorname{det} V_{4}^{(3,4,1)}}{\operatorname{det} V_{4}^{(3)}}=-\frac{\operatorname{det} V_{4}^{(3,3,4)}}{\operatorname{det} V_{4}^{(3)}} \quad \text { for } n \geq 1, \\
P-J(n)=\frac{\operatorname{det} V_{4}^{(4,4,1)}}{\operatorname{det} V_{4}^{(4)}}=-\frac{\operatorname{det} V_{4}^{(4,3,4)}}{2 \cdot \operatorname{det} V_{4}^{(4)}} \quad \text { for } n \geq 1, \\
P a-P(n)=\frac{\operatorname{det} V_{5}^{(5,5,1)}}{\operatorname{det} V_{5}^{(5)}}=-\frac{\operatorname{det} V_{5}^{(5,4,5)}}{\operatorname{det} V_{5}^{(5)}} \quad \text { for } n \geq 6
\end{gathered}
$$

and

$$
P a-J(n)=\frac{\operatorname{det} V_{5}^{(6,5,1)}}{\operatorname{det} V_{5}^{(6)}}=-\frac{\operatorname{det} V_{5}^{(6,4,5)}}{2 \cdot \operatorname{det} V_{5}^{(6)}} \quad \text { for } n \geq 4
$$

\subsection{Generating functions}

It is easy to see that the generating functions of the sequences $\{F-J(n)\},\{P a-F(n)\}$, $\{P-F(n)\},\{P-J(n)\},\{P a-P(n)\}$ and $\{P a-J(n)\}$ are, respectively,

$$
\begin{array}{ll}
g_{1}(x)=\frac{x^{3}}{1-2 x-2 x^{2}+3 x^{3}+2 x^{4}}, & \left(0 \leq 2 x+2 x^{2}-3 x^{3}-2 x^{4}<1\right), \\
g_{2}(x)=\frac{x^{4}}{1-x-2 x^{2}+2 x^{4}+x^{5}}, & \left(0 \leq x+2 x^{2}-2 x^{4}-x^{5}<1\right), \\
g_{3}(x)=\frac{x^{3}}{1-3 x+3 x^{3}+x^{4}}, & \left(0 \leq 3 x-3 x^{3}-x^{4}<1\right), \\
g_{4}(x)=\frac{x^{3}}{1-3 x-x^{2}+5 x^{3}+2 x^{4}}, & \left(0 \leq 3 x+x^{2}-5 x^{3}-2 x^{4}<1\right), \\
g_{5}(x)=\frac{x^{4}}{1-2 x-2 x^{2}+x^{3}+3 x^{4}+x^{5}}, & \left(0 \leq 2 x+2 x^{2}-x^{3}-3 x^{4}-x^{5}<1\right)
\end{array}
$$

and

$$
g_{6}(x)=\frac{x^{4}}{1-x-3 x^{2}+3 x^{4}+2 x^{5}}, \quad\left(0 \leq x+3 x^{2}-3 x^{4}-2 x^{5}<1\right)
$$




\subsection{Exponential representations}

Now considering the functions $g_{1}(x), g_{2}(x), g_{3}(x), g_{4}(x), g_{5}(x)$ and $g_{6}(x)$, we can give the exponential representations for the Fibonacci-Jacobsthal, Padovan-Fibonacci, Pell-Fibonacci, Pell-Jacobsthal, Padovan-Pell, and Padovan-Jacobsthal numbers by the following Theorem.

Theorem 2.3. The sequences $\{F-J(n)\},\{P a-F(n)\},\{P-F(n)\},\{P-J(n)\},\{P a-P(n)\}$ and $\{P a-J(n)\}$ have the following exponential representations, respectively:

$$
\begin{aligned}
& g_{1}(x)=x^{3} \exp \left(\sum_{i=1}^{\infty} \frac{x^{i}}{i}\left(2+2 x-3 x^{2}-2 x^{3}\right)^{i}\right), \\
& g_{2}(x)=x^{4} \exp \left(\sum_{i=1}^{\infty} \frac{x^{i}}{i}\left(1+2 x-2 x^{3}-x^{4}\right)^{i}\right), \\
& g_{3}(x)=x^{3} \exp \left(\sum_{i=1}^{\infty} \frac{x^{i}}{i}\left(3-3 x^{2}-x^{3}\right)^{i}\right), \\
& g_{4}(x)=x^{3} \exp \left(\sum_{i=1}^{\infty} \frac{x^{i}}{i}\left(3+x-5 x^{2}-2 x^{3}\right)^{i}\right), \\
& g_{5}(x)=x^{4} \exp \left(\sum_{i=1}^{\infty} \frac{x^{i}}{i}\left(2+2 x-x^{2}-3 x^{3}-x^{4}\right)^{i}\right)
\end{aligned}
$$

and

$$
g_{6}(x)=x^{4} \exp \left(\sum_{i=1}^{\infty} \frac{x^{i}}{i}\left(1+3 x-3 x^{3}-2 x^{4}\right)^{i}\right) .
$$

Proof. Consider the sequence $\{P-F(n)\}$. Since $\ln \frac{g_{3}(x)}{x^{3}}=-\ln \left(1-3 x+3 x^{3}+x^{4}\right)$ and $\ln \left(1-3 x+3 x^{3}+x^{4}\right)=-\left[x\left(3-3 x^{2}-x^{3}\right)+\frac{1}{2} x^{2}\left(3-3 x^{2}-x^{3}\right)^{2}+\cdots+\frac{1}{i} x^{i}\left(3-3 x^{2}-x^{3}\right)^{i}\right]$, by a simple calculation, we obtain the conclusion.

There are similar proofs for other sequences.

\subsection{Combinatorial representations}

Here we investigate the combinatorial representations for the Fibonacci-Jacobsthal, PadovanFibonacci, Pell-Fibonacci, Pell-Jacobsthal, Padovan-Pell, and Padovan-Jacobsthal numbers.

Let $K\left(k_{1}, k_{2}, \ldots, k_{v}\right)$ be a $v \times v$ companion matrix as follows:

$$
K\left(k_{1}, k_{2}, \ldots, k_{v}\right)=\left[\begin{array}{cccc}
k_{1} & k_{2} & \cdots & k_{v} \\
1 & 0 & \cdots & 0 \\
\vdots & \ddots & \ddots & \vdots \\
0 & \cdots & 1 & 0
\end{array}\right]
$$

For more details on the companion type matrices, see $[18,19]$. 
Theorem 2.4. (Chen and Louck [3]). The $(i, j)$ entry $k_{i, j}^{(n)}\left(k_{1}, k_{2}, \ldots, k_{v}\right)$ in the matrix $K^{n}\left(k_{1}, k_{2}, \ldots, k_{v}\right)$ is given by the following formula:

$$
k_{i, j}^{(n)}\left(k_{1}, k_{2}, \ldots, k_{v}\right)=\sum_{\left(t_{1}, t_{2}, \ldots, t_{v}\right)} \frac{t_{j}+t_{j+1}+\cdots+t_{v}}{t_{1}+t_{2}+\cdots+t_{v}} \times\left(\begin{array}{c}
t_{1}+\cdots+t_{v} \\
t_{1}, \ldots, t_{v}
\end{array}\right) k_{1}^{t_{1}} \cdots k_{v}^{t_{v}},
$$

where the summation is over nonnegative integers satisfying $t_{1}+2 t_{2}+\cdots+v t_{v}=n-i+j$, $\left(\begin{array}{c}t_{1}+\cdots+t_{v} \\ t_{1}, \ldots, t_{v}\end{array}\right)=\frac{\left(t_{1}+\cdots+t_{v}\right) !}{t_{1} ! \cdots t_{v} !}$ is a multinomial coefficient, and the coefficients in (7) are defined to be 1 if $n=i-j$.

Then we have the following Corollary.

Corollary 2.2. $\quad$ (i) $F-J(n)=\sum_{\left(t_{1}, t_{2}, t_{3}, t_{4}\right)}\left(\begin{array}{c}t_{1}+t_{2}+t_{3}+t_{4} \\ t_{1}, t_{2}, t_{3}, t_{4}\end{array}\right) 2^{t_{1}+t_{2}}(-3)^{t_{3}}(-2)^{t_{4}},(n \geq 3)$ where the summation is over nonnegative integers satisfying $t_{1}+2 t_{2}+3 t_{3}+4 t_{4}=n-3$.

(ii) $P a-F(n)=\sum_{\left(t_{1}, t_{2}, t_{3}, t_{4}, t_{5}\right)}\left(\begin{array}{c}t_{1}+t_{2}+t_{3}+t_{4}+t_{5} \\ t_{1}, t_{2}, t_{3}, t_{4}, t_{5}\end{array}\right) 2^{t_{2}}(-2)^{t_{4}}(-1)^{t_{5}},(n \geq 5)$

where the summation is over nonnegative integers satisfying $t_{1}+2 t_{2}+3 t_{3}+4 t_{4}+5 t_{5}=n-4$ and

$P a-F(n)=-\left(\sum_{\left(t_{1}, t_{2}, t_{3}, t_{4}, t_{5}\right)} \frac{t_{5}}{t_{1}+t_{2}+t_{3}+t_{4}+t_{5}} \times\left(\begin{array}{c}t_{1}+t_{2}+t_{3}+t_{4}+t_{5} \\ t_{1}, t_{2}, t_{3}, t_{4}, t_{5}\end{array}\right) 2^{t_{2}}(-2)^{t_{4}}(-1)^{t_{5}}\right),(n \geq 5)$ where the summation is over nonnegative integers satisfying $t_{1}+2 t_{2}+3 t_{3}+4 t_{4}+5 t_{5}=n+1$.

(iii) $P-F(n)=\sum_{\left(t_{1}, t_{2}, t_{3}, t_{4}\right)}\left(\begin{array}{c}t_{1}+t_{2}+t_{3}+t_{4} \\ t_{1}, t_{2}, t_{3}, t_{4}\end{array}\right) 3^{t_{1}}(-3)^{t_{3}}(-1)^{t_{4}},(n \geq 1)$

where the summation is over nonnegative integers satisfying $t_{1}+2 t_{2}+3 t_{3}+4 t_{4}=n-3$ and $P-F(n)=-\left(\sum_{\left(t_{1}, t_{2}, t_{3}, t_{4}\right)} \frac{t_{4}}{t_{1}+t_{2}+t_{3}+t_{4}}\left(\begin{array}{c}t_{1}+t_{2}+t_{3}+t_{4} \\ t_{1}, t_{2}, t_{3}, t_{4}\end{array}\right) 3^{t_{1}}(-3)^{t_{3}}(-1)^{t_{4}}\right),(n \geq 1)$

where the summation is over nonnegative integers satisfying $t_{1}+2 t_{2}+3 t_{3}+4 t_{4}=n+1$.

(iv) $P-J(n)=\sum_{\left(t_{1}, t_{2}, t_{3}, t_{4}\right)}\left(\begin{array}{c}t_{1}+t_{2}+t_{3}+t_{4} \\ t_{1}, t_{2}, t_{3}, t_{4}\end{array}\right) 3^{t_{1}}(-5)^{t_{3}}(-2)^{t_{4}},(n \geq 1)$

where the summation is over nonnegative integers satisfying $t_{1}+2 t_{2}+3 t_{3}+4 t_{4}=n-3$ and

$$
P-J(n)=-\frac{1}{2}\left(\sum_{\left(t_{1}, t_{2}, t_{3}, t_{4}\right)} \frac{t_{4}}{t_{1}+t_{2}+t_{3}+t_{4}}\left(\begin{array}{c}
t_{1}+t_{2}+t_{3}+t_{4} \\
t_{1}, t_{2}, t_{3}, t_{4}
\end{array}\right) 3^{t_{1}}(-5)^{t_{3}}(-2)^{t_{4}}\right),(n \geq 1)
$$

where the summation is over nonnegative integers satisfying $t_{1}+2 t_{2}+3 t_{3}+4 t_{4}=n+1$. 
(v) $P a-P(n)=\sum_{\left(t_{1}, t_{2}, t_{3}, t_{4}, t_{5}\right)}\left(\begin{array}{c}t_{1}+t_{2}+t_{3}+t_{4}+t_{5} \\ t_{1}, t_{2}, t_{3}, t_{4}, t_{5}\end{array}\right) 2^{t_{1}+t_{2}}(-1)^{t_{3}+t_{5}}(-3)^{t_{4}},(n \geq 6)$

where the summation is over nonnegative integers satisfying $t_{1}+2 t_{2}+3 t_{3}+4 t_{4}+5 t_{5}=n-4$

and

$P a-P(n)=-\left(\sum_{\left(t_{1}, t_{2}, t_{3}, t_{4}, t_{5}\right)} \frac{t_{5}}{t_{1}+t_{2}+t_{3}+t_{4}+t_{5}} \times\left(\begin{array}{c}t_{1}+t_{2}+t_{3}+t_{4}+t_{5} \\ t_{1}, t_{2}, t_{3}, t_{4}, t_{5}\end{array}\right) 2^{t_{1}+t_{2}}(-1)^{t_{3}+t_{5}}(-3)^{t_{4}}\right),(n \geq 6)$

where the summation is over nonnegative integers satisfying $t_{1}+2 t_{2}+3 t_{3}+4 t_{4}+5 t_{5}=n+1$.

(vi) $P a-J(n)=\sum_{\left(t_{1}, t_{2}, t_{3}, t_{4}, t_{5}\right)}\left(\begin{array}{c}t_{1}+t_{2}+t_{3}+t_{4}+t_{5} \\ t_{1}, t_{2}, t_{3}, t_{4}, t_{5}\end{array}\right) 3^{t_{2}}(-3)^{t_{4}}(-2)^{t_{5}},(n \geq 4)$

where the summation is over nonnegative integers satisfying $t_{1}+2 t_{2}+3 t_{3}+4 t_{4}+5 t_{5}=n-4$

and

$P a-J(n)=-\frac{1}{2}\left(\sum_{\left(t_{1}, t_{2}, t_{3}, t_{4}, t_{5}\right)} \frac{t_{5}}{t_{1}+t_{2}+t_{3}+t_{4}+t_{5}} \times\left(\begin{array}{c}t_{1}+t_{2}+t_{3}+t_{4}+t_{5} \\ t_{1}, t_{2}, t_{3}, t_{4}, t_{5}\end{array}\right) 3^{t_{2}}(-3)^{t_{4}}(-2)^{t_{5}}\right),(n \geq 4)$

where the summation is over nonnegative integers satisfying $t_{1}+2 t_{2}+3 t_{3}+4 t_{4}+5 t_{5}=n+1$.

Proof. Consider the case (iv). If we take $i=4, j=1$ for first case and $i=3, j=4$ for second case in Theorem 2.4, then we can directly see the conclusions from $\left(M_{4}\right)^{n}$.

There are similar proofs for the sequences $\{F-J(n)\},\{P a-F(n)\},\{P-F(n)\},\{P a-P(n)\}$ and $\{P a-J(n)\}$.

\subsection{Permanental representations}

Now we concentrate on finding the permanental representations of defined these sequences.

Definition 2.1. A $u \times v$ real matrix $M=\left[m_{i, j}\right]$ is called a contractible matrix in the $k$-th column (respectively, row) if the $k$-th column (respectively, row) contains exactly two non-zero entries.

Suppose that $x_{1}, x_{2}, \ldots, x_{u}$ are row vectors of the matrix $M$. If $M$ is contractible in the $k$-th column such that $m_{i, k} \neq 0, m_{j, k} \neq 0$ and $i \neq j$, then the $(u-1) \times(v-1)$ matrix $M_{i j: k}$ obtained from $M$ by replacing the $i$-th row with $m_{i, k} x_{j}+m_{j, k} x_{i}$ and deleting the $j$-th row. The $k$-th column is called the contraction in the $k$-th column relative to the $i$-th row and the $j$-th row.

In [2], Brualdi and Gibson obtained that $\operatorname{per}(M)=\operatorname{per}(N)$ if $M$ is a real matrix of order $\alpha>1$ and $N$ is a contraction of $M$.

Now we consider the relationships among the Fibonacci-Jacobsthal, Padovan-Fibonacci, Pell-Fibonacci, Pell-Jacobsthal, Padovan-Pell and, Padovan-Jacobsthal numbers and the permanents of the certain matrices which are obtained by using the generating matrix of the Fibonacci-Jacobsthal, Padovan-Fibonacci, Pell-Fibonacci, Pell-Jacobsthal, Padovan-Pell, and Padovan-Jacobsthal numbers. Let $K^{(1)}(m)=\left[k_{i, j}^{(1)}\right], K^{(2)}(m)=\left[k_{i, j}^{(2)}\right], K^{(3)}(m)=\left[k_{i, j}^{(3)}\right]$, $K^{(4)}(m)=\left[k_{i, j}^{(4)}\right], K^{(5)}(m)=\left[k_{i, j}^{(5)}\right]$ and $K^{(6)}(m)=\left[k_{i, j}^{(6)}\right]$ be the $m \times m$ super-diagonal matrices as follows, respectively: 


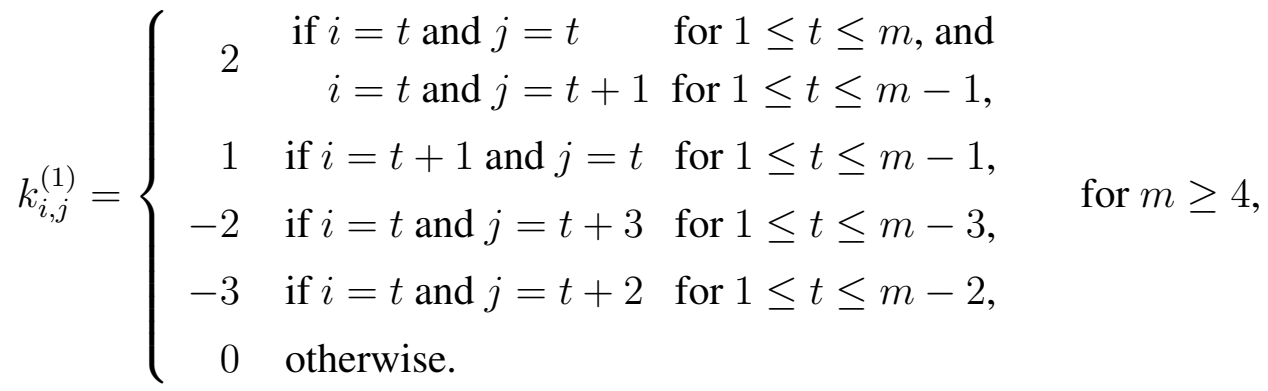

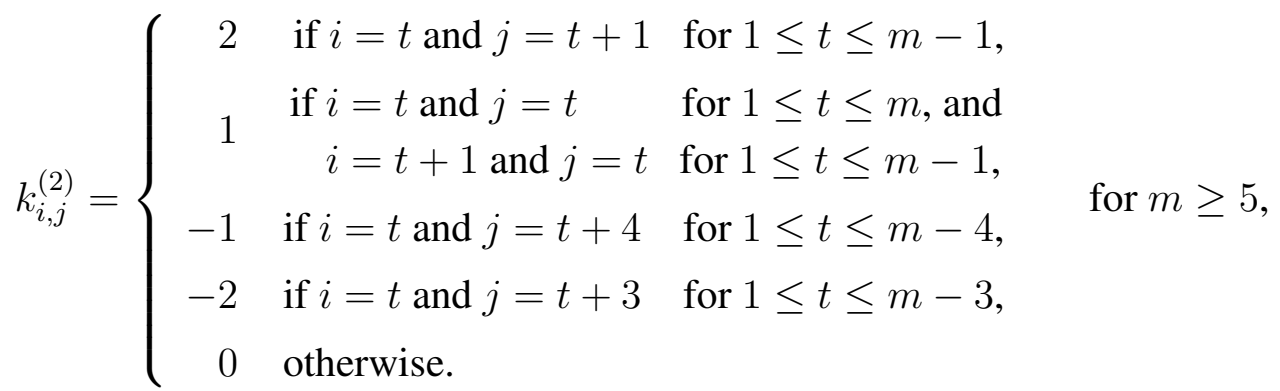

$$
\begin{aligned}
& k_{i, j}^{(3)}=\left\{\begin{array}{rll}
3 & \text { if } i=t \text { and } j=t \quad \text { for } 1 \leq t \leq m, \\
1 & \text { if } i=t+1 \text { and } j=t \quad \text { for } 1 \leq t \leq m-1, \\
-1 & \text { if } i=t \text { and } j=t+3 \text { for } 1 \leq t \leq m-3, \\
-3 & \text { if } i=t \text { and } j=t+2 \text { for } 1 \leq t \leq m-2, \\
0 & \text { otherwise. }
\end{array} \quad \text { for } m \geq 4,\right.
\end{aligned}
$$

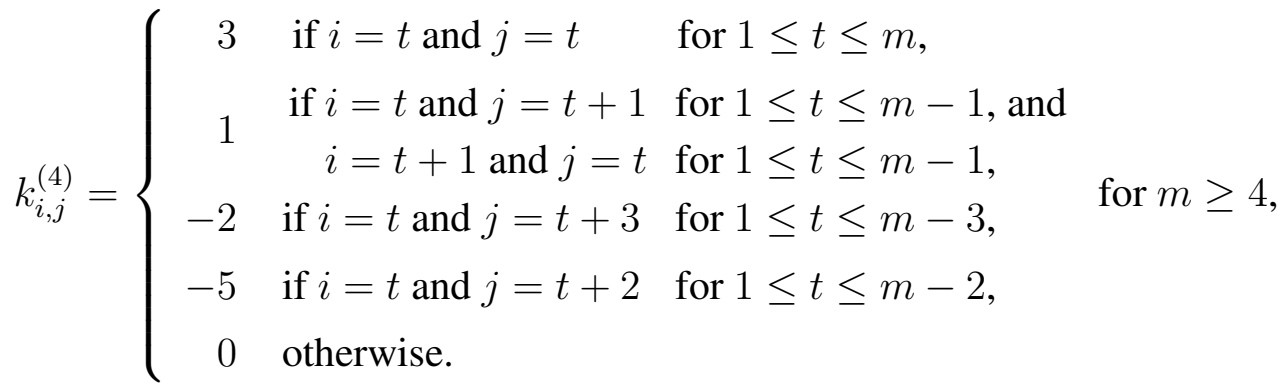

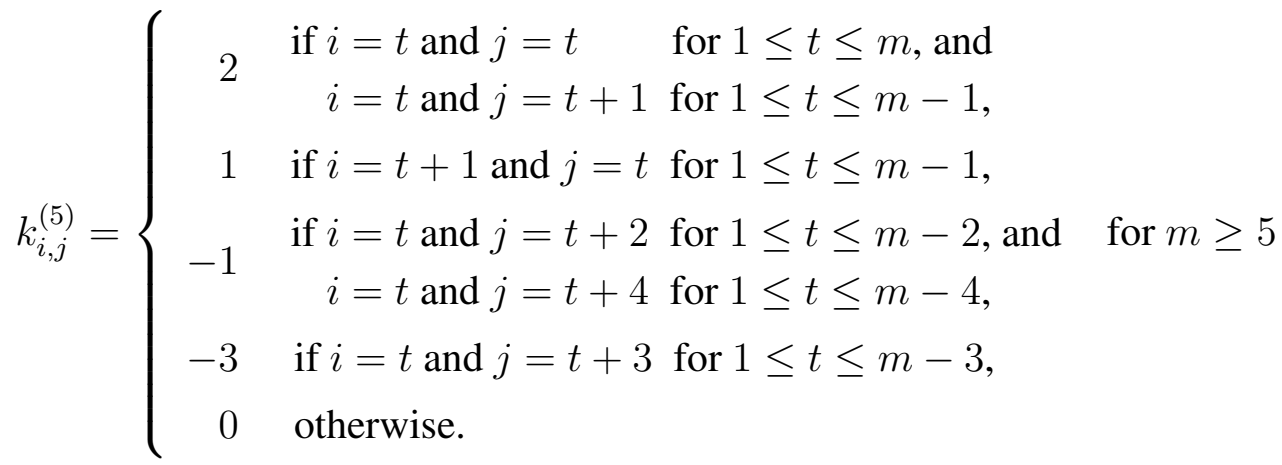

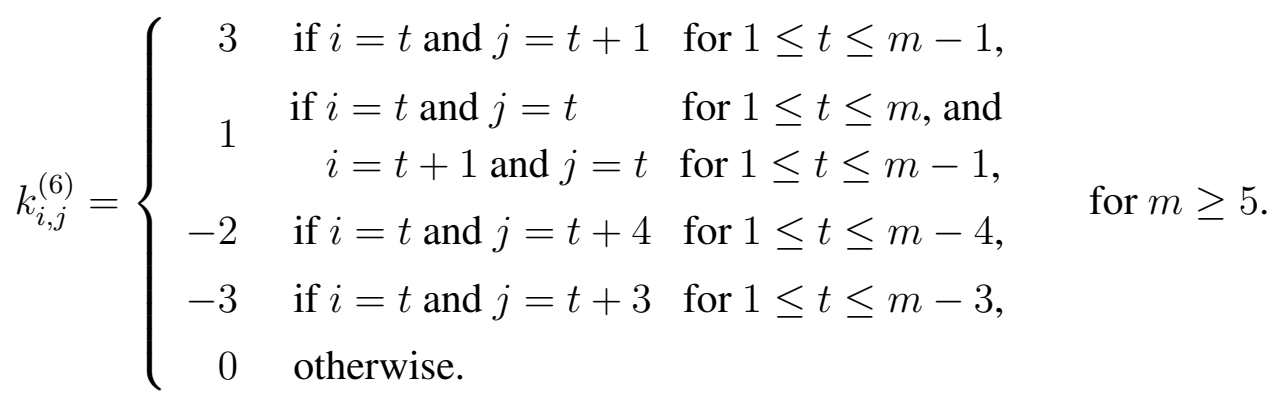


Then we have the following Theorem.

Theorem 2.5. (i). For $m \geqslant 4$ and $k=1,3,4$,

$$
\operatorname{per} K^{(k)}(m)=x_{m+3}^{k} \text {. }
$$

(ii). For $m \geqslant 5$ and $k=2,5,6$,

$$
\operatorname{per} K^{(k)}(m)=x_{m+4}^{k} \text {. }
$$

Proof. Consider the subcase of $k=3$ in case (i). Let us consider matrix $K^{(3)}(m)$ and let the equation be holds for $m \geqslant 4$. Then we show that the equation holds for $m+1$. If we expand the per $K^{(3)}(m)$ by the Laplace expansion of permanent with respect to the first row, then we obtain

$$
\operatorname{per} K^{(3)}(m+1)=3 \text {. per } K^{(3)}(m)-3 \text {. per } K^{(3)}(m-2)-\operatorname{per} K^{(3)}(m-3) \text {. }
$$

Since per $K^{(3)}(m)=x_{m+3}^{3}$, per $K^{(3)}(m-2)=x_{m+1}^{3}$ and per $K^{(3)}(m-3)=x_{m}^{3}$, we easily obtain per $K^{(3)}(m+1)=x_{m+4}^{3}$. So the proof is complete.

There are similar proofs for other matrices.

Let $L^{(1)}(m)=\left[l_{i, j}^{(1)}\right], L^{(2)}(m)=\left[l_{i, j}^{(2)}\right], L^{(3)}(m)=\left[l_{i, j}^{(3)}\right], L^{(4)}(m)=\left[l_{i, j}^{(4)}\right], L^{(5)}(m)=$ $\left[l_{i, j}^{(5)}\right]$ and $L^{(6)}(m)=\left[l_{i, j}^{(6)}\right]$ be the $m \times m$ matrices as follows, respectively:

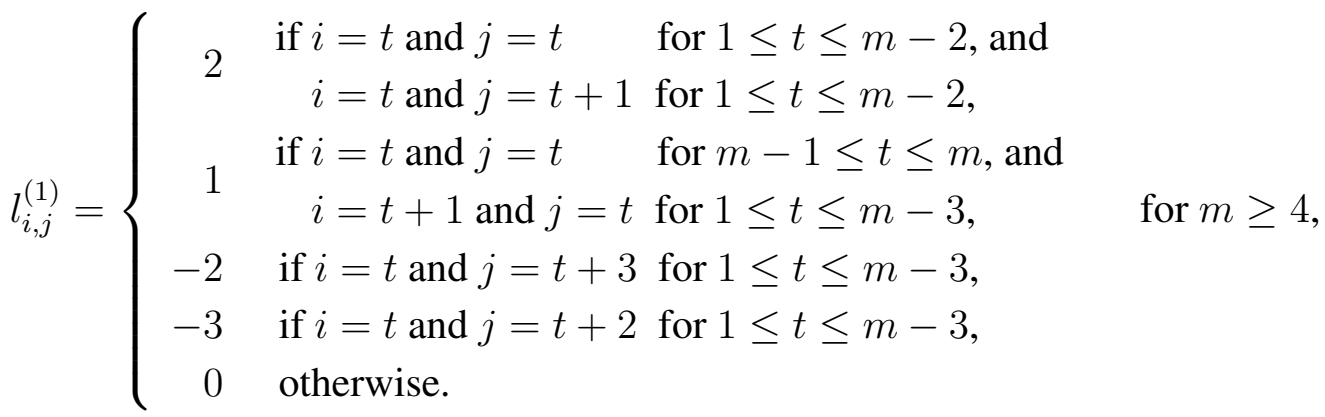

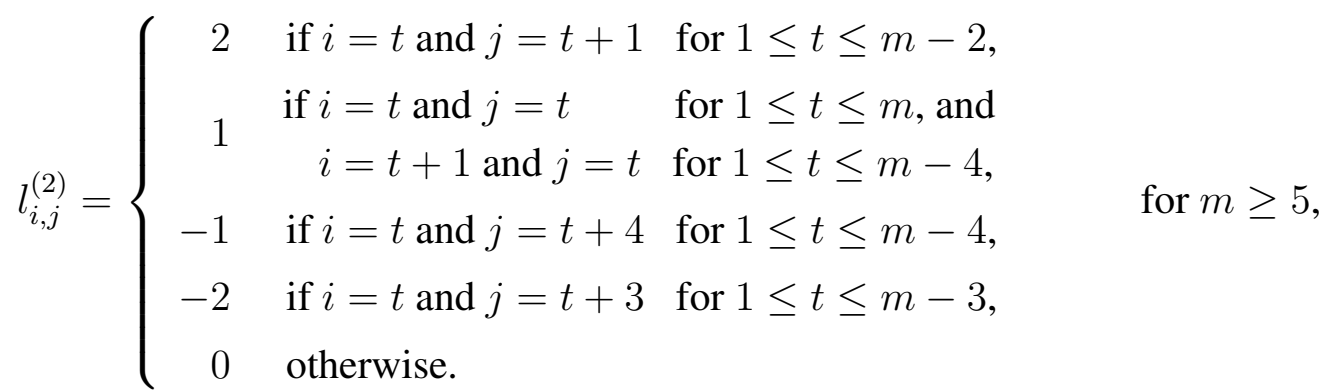

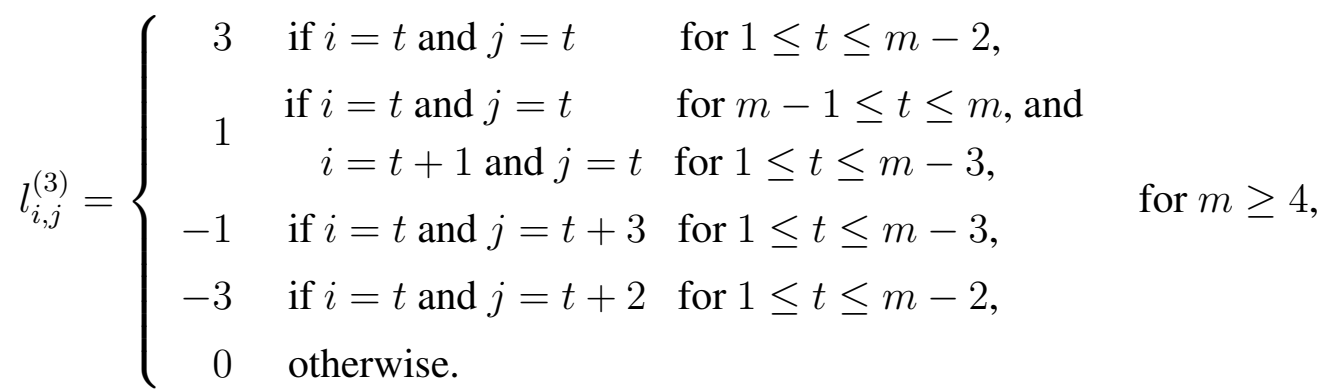




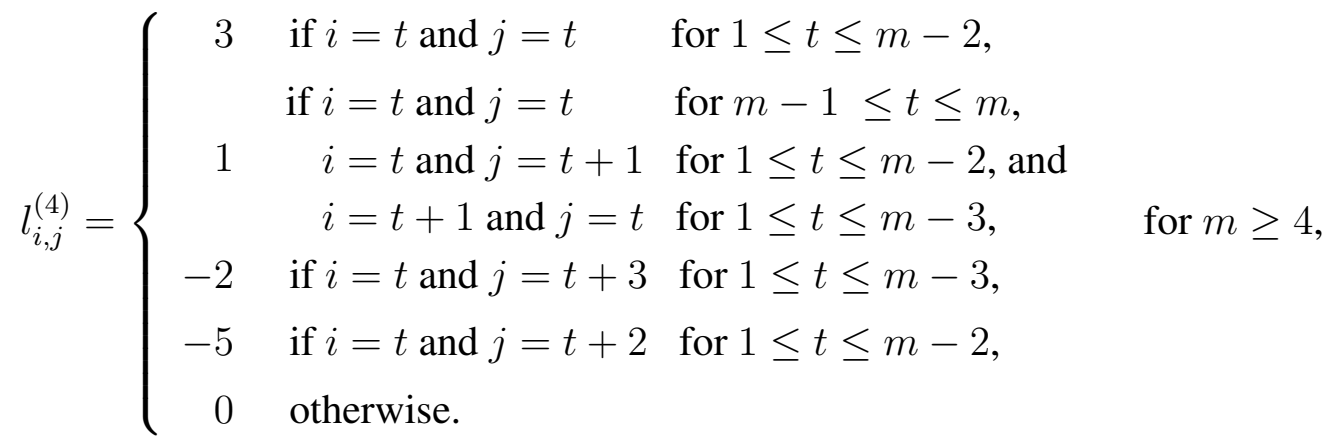

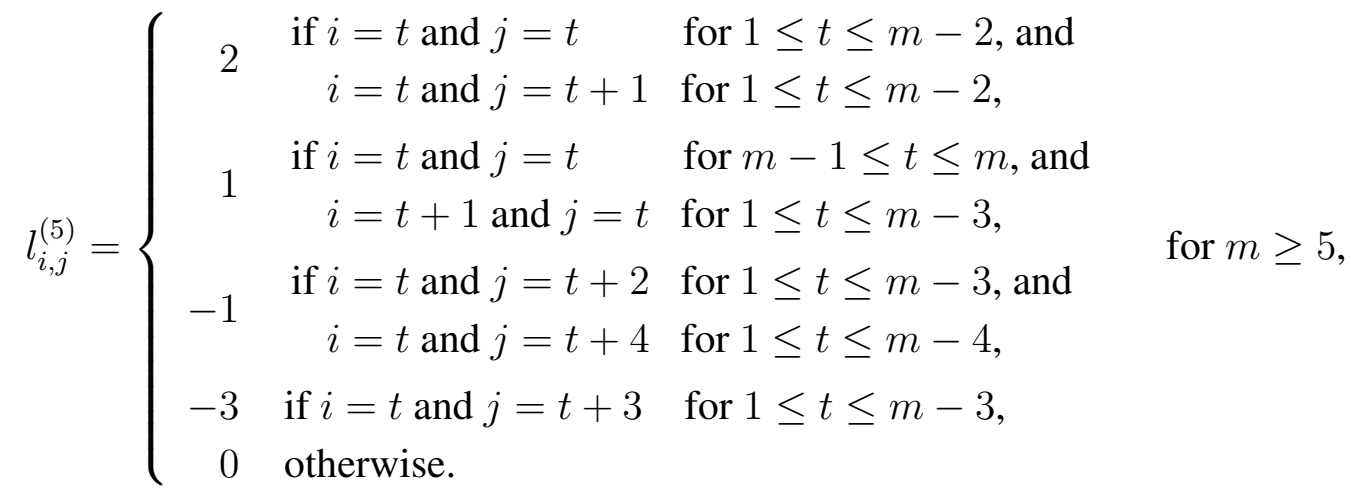

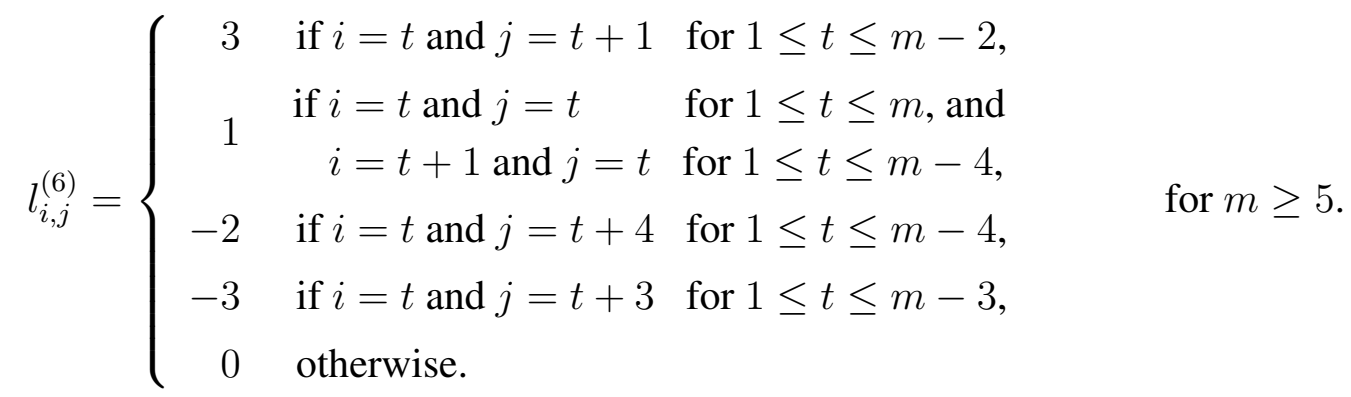

Then we have the following Theorem.

Theorem 2.6. (i). For $m \geqslant 4$ and $k=1,3,4$,

$$
\operatorname{per} L^{(k)}(m)=x_{m+1}^{k} \text {. }
$$

(ii). For $m \geqslant 5$ and $k=2,6$,

$$
\operatorname{per} L^{(k)}(m)=x_{m+1}^{k} \text {. }
$$

(iii). For $m \geqslant 5$,

$$
\operatorname{per} L^{(5)}(m)=x_{m+2}^{5} \text {. }
$$

Proof. Consider the subcase of $k=6$ in case (ii). Let us consider the matrix $L^{(6)}(m)$ and let the equation holds for $m \geq 5$. Then we show that the equation holds for $m+1$. If we expand per $L^{(6)}(m)$ by the Laplace expansion of permanent according to the first row, then we obtain:

per $L^{(6)}(m+1)=\operatorname{per} L^{(6)}(m)+3 \operatorname{per} L^{(6)}(m-1)-3 \operatorname{per} L^{(6)}(m-3)-2 \operatorname{per} L^{(6)}(m-4)$.

Also, since per $L^{(6)}(m)=x_{m+1}^{6}$, per $L^{(6)}(m-1)=x_{m}^{6}$, per $L^{(6)}(m-3)=x_{m-2}^{6}$ and per $L^{(6)}(m-4)=x_{m-3}^{6}$, it is clear that per $L^{(6)}(m+1)=x_{m+2}^{6}$.

There are similar proofs for other matrices. 
Assume that $N^{(1)}(m)=\left[n_{i, j}^{(1)}\right], N^{(2)}(m)=\left[n_{i, j}^{(2)}\right], N^{(3)}(m)=\left[n_{i, j}^{(3)}\right], N^{(4)}(m)=\left[n_{i, j}^{(4)}\right]$, $N^{(5)}(m)=\left[n_{i, j}^{(5)}\right]$ and $N^{(6)}(m)=\left[n_{i, j}^{(6)}\right]$ are the $m \times m$ matrices as shown, respectively:

$$
\begin{aligned}
& (m-2) \text {-th } \\
& N^{(k)}(m)=\left[\begin{array}{ccccc}
1 & \cdots & 1 & 0 & 0 \\
1 & & & & \\
0 & & & \\
\vdots & & & L^{(k)}(m-1)
\end{array}\right] \text {, for } m>4 \text { and } k=1,4, \\
& (m-3)-\text { th } \\
& N^{(k)}(m)=\left[\begin{array}{cccccc}
1 & \cdots & 1 & 0 & 0 & 0 \\
1 & & & & & \\
0 & & & & \\
\vdots & & & L^{(k)}(m-1) & \\
0 & & &
\end{array}\right] \text {, for } m>5 \text { and } k=2,6 \\
& (m-3)-\text { th } \\
& N^{(3)}(m)=\left[\begin{array}{cccccc}
1 & \cdots & 1 & 0 & 0 & 0 \\
1 & & & & & \\
0 & & & \\
\vdots & & & L^{(3)}(m-1) & \\
0 & & &
\end{array}\right], \text { for } m>4
\end{aligned}
$$

and

$$
N^{(5)}(m)=\left[\begin{array}{ccccc}
1 & \cdots & 1 & 0 & 0 \\
1 & & & & \\
0 & & & \\
\vdots & & & L^{(5)}(m-1) \\
0 & & &
\end{array}\right], \text { for } m>5
$$

then we have the following results:

Theorem 2.7. (i). For $m>4$ and $k=1,3,4$,

$$
\operatorname{per} N^{(k)}(m)=\sum_{i=0}^{m} x_{i}^{k} \text {. }
$$

(ii). For $m>5$ and $k=2,6$,

$$
\operatorname{per} N^{(k)}(m)=\sum_{i=0}^{m} x_{i}^{k} \text {. }
$$


(iii). For $m>5$,

$$
\operatorname{per} N^{(5)}(m)=\sum_{i=0}^{m+1} x_{i}^{5}
$$

Proof. Consider the subcase of $k=6$ in case (ii). If we extend per $N^{(6)}(m)$ with respect to the first row, we write

$$
\operatorname{per} N^{(6)}(m)=\operatorname{per} N^{(6)}(m-1)+\operatorname{per} L^{(6)}(m-1) .
$$

Thus, by the results and an inductive argument, the proof is easily seen.

\subsection{Certain related determinants}

A matrix $M$ is called convertible if there is an $n \times n(1,-1)$-matrix $K$ such that per $M=$ $\operatorname{det}(M \circ K)$, where $M \circ K$ denotes the Hadamard product of $M$ and $K$.

Now we give relationships among the Fibonacci-Jacobsthal, Padovan-Fibonacci, PellFibonacci, Pell-Jacobsthal, Padovan-Pell and Padovan-Jacobsthal numbers, and the determinants of the certain matrices which are obtained by using the matrices $K^{(k)}(m), L^{(k)}(m)$ and $N^{(k)}(m)$. Let $k=1,2, \ldots, 6$ and let $R$ be the $m \times m$ matrix, defined by

$$
R=\left[\begin{array}{cccccc}
1 & 1 & 1 & \cdots & 1 & 1 \\
-1 & 1 & 1 & \cdots & 1 & 1 \\
1 & -1 & 1 & \cdots & 1 & 1 \\
\vdots & \ddots & \vdots & \ddots & \vdots & \vdots \\
1 & \cdots & 1 & -1 & 1 & 1 \\
1 & \cdots & 1 & 1 & -1 & 1
\end{array}\right]
$$

Corollary 2.3. (i).

$$
\operatorname{det}\left(K^{(k)}(m) \circ R\right)=x_{m+3}^{k}, \text { for } m \geq 4 \text { and } k=1,3,4
$$

and

$$
\operatorname{det}\left(K^{(k)}(m) \circ R\right)=x_{m+4}^{k}, \text { for } m \geq 5 \text { and } k=2,5,6 \text {. }
$$

(ii).

$$
\begin{aligned}
& \operatorname{det}\left(L^{(k)}(m) \circ R\right)=x_{m+1}^{k}, \text { for } m \geq 4 \text { and } k=1,3,4, \\
& \operatorname{det}\left(L^{(k)}(m) \circ R\right)=x_{m+1}^{k}, \text { for } m \geq 5 \text { and } k=2,6
\end{aligned}
$$

and

$$
\operatorname{det}\left(L^{(5)}(m) \circ R\right)=x_{m+2}^{5}, \text { for } m \geq 5 \text {. }
$$

(iii).

$$
\begin{aligned}
& \operatorname{det}\left(N^{(k)}(m) \circ R\right)=\sum_{i=0}^{m} x_{i}^{k}, \text { for } m>4 \text { and } k=1,3,4, \\
& \operatorname{det}\left(N^{(2)}(m) \circ R\right)=\sum_{i=0}^{m} x_{i}^{k}, \text { for } m>5 \text { and } k=2,6
\end{aligned}
$$

and

$$
\operatorname{det}\left(N^{(5)}(m) \circ R\right)=\sum_{i=0}^{m+1} x_{i}^{5}, \text { for } m>5 \text {. }
$$


Proof. Since per $K^{(k)}(m)=\operatorname{det}\left(K^{(k)}(m) \circ R\right)$, per $L^{(k)}(m)=\operatorname{det}\left(L^{(k)}(m) \circ R\right)$ and per $N^{(k)}(m)=\operatorname{det}\left(N^{(k)}(m) \circ R\right)$ for $k=1,2, \ldots, 6$, by Theorem 2.5, Theorem 2.6 and Theorem 2.7, we have the conclusion.

\subsection{Related sums}

Now we consider the sums of the Fibonacci-Jacobsthal, Padovan-Fibonacci, Pell-Fibonacci, Pell-Jacobsthal, Padovan-Pell and Padovan-Jacobsthal numbers. Let

$$
S_{n}=\sum_{i=0}^{n} x_{i}^{k}
$$

for $n \geq 1$ and let $T_{1}^{k}$ and $T_{2}^{k}$ be the $5 \times 5$ and $6 \times 6$ matrices as shown, respectively:

$$
T_{1}^{k}=\left[\begin{array}{ccccc}
1 & 0 & 0 & 0 & 0 \\
1 & & & & \\
0 & & & & \\
0 & & & M_{k} & \\
0 & & & &
\end{array}\right], \text { for } k=1,3,4
$$

and

$$
T_{2}^{k}=\left[\begin{array}{cccccc}
1 & 0 & 0 & 0 & 0 & 0 \\
1 & & & & & \\
0 & & & & & \\
0 & & & M_{k} & & \\
0 & & & & \\
0 & & & & &
\end{array}\right], \text { for } k=2,5,6 .
$$

If we use induction on $n$, then we obtain

$$
\left(T_{1}^{k}\right)^{n}=\left[\begin{array}{ccccc}
1 & 0 & 0 & 0 & 0 \\
S_{n+2} & & & \\
S_{n+1} & & & \\
S_{n} & & & \left(M_{k}\right)^{n} & \\
S_{n-1} & & &
\end{array}\right], \text { for } k=1,3,4,
$$

and

$$
\left(T_{2}^{k}\right)^{n}=\left[\begin{array}{cccccc}
1 & 0 & 0 & 0 & 0 & 0 \\
S_{n+3} & & & & & \\
S_{n+2} & & & & \\
S_{n+1} & & & \left(M_{k}\right)^{n} & & \\
S_{n} & & & & \\
S_{n-1} & & & & &
\end{array}\right], \text { for } k=2,5,6
$$




\section{References}

[1] Bradie, B. (2010). Extension and refinements of some properties of sums involving Pell number. Missouri Journal of Mathematical Sciences, 22(1), 37-43.

[2] Brualdi, R. A., \& Gibson, P. M. (1977). Convex polyhedra of doubly stochastic matrices. I. Applications of permanent function. Journal of Combinatorial Theory, Series A, 22(2), 194-230.

[3] Chen, W. Y. C., \& Louck, J. D. (1996). The combinatorial power of the companion matrix. Linear Algebra and its Applications, 232, 261-278.

[4] Devaney, R. L. (1999). The Mandelbrot set, the Farey tree, and the Fibonacci sequence. American Mathematical Monthly, 106(4), 289-302.

[5] Deveci, Ö. (2018). The Padovan-circulant sequences and their applications. Mathematical Reports, 20(70), 401-416.

[6] Deveci, Ö., \& Artun, G. (2019). On the adjacency-Jacobsthal numbers. Communications in Algebra, 47(11), 4520-4532.

[7] Deveci, Ö., \& Karaduman, E. (2017). On the Padovan p-numbers. Hacettepe Journal of Mathematics and Statistics, 46(4), 579-592.

[8] Frey, D. D., \& Sellers, J. A. (2000) Jacobsthal numbers and alternating sign matrices. Journal of Integer Sequences, 3, Article 00.2.3.

[9] Gogin, N. D., \& Myllari, A. A. (2007). The Fibonacci-Padovan sequence and MacWilliams transform matrices. Programming and Computer Software, 33(2), 74-79.

[10] Horadam, A. F. (1996). Jacobsthal representations numbers, The Fibonacci Quarterly, $34(1), 40-54$.

[11] Horadam, A. F. (1994). Applications of modified Pell numbers to representations. Ulam Quarterly, 3(1), 34-53.

[12] Johnson, R. C. (2009). Fibonacci Numbers and Matrices. Available online at: https: //maths.dur.ac.uk/ dma0rcj/PED/fib.pdf.

[13] Kalman, D. (1982). Generalized Fibonacci numbers by matrix methods. The Fibonacci Quarterly, 20(1), 73-76.

[14] Kilic, E. (2009). The generalized Pell ( $p, i)$-numbers and their Binet formulas, combinatorial representations, sums. Chaos, Solitons \& Fractals, 40(4), 2047-2063.

[15] Kilic, E., \& Tasci, D. (2006). The generalized Binet formula, representation and sums of the generalized order- $k$ Pell numbers. Taiwanese Journal of Mathematics, 10(6), 1661-1670. 
[16] Kocer, E. G., \& Tuglu N. (2007). The Binet formulas for the Pell and Pell-Lucas $p$-numbers. Ars Combinatoria, 85, 3-17.

[17] Koken, F., \& Bozkurt, D. (2008). On the Jacobsthal numbers by matrix methods. International Journal of Contemporary Mathematical Sciences, 3(13), 605-614.

[18] Lancaster, P. \& Tismenetsky, M. (1985) The Theory of Matrices: With Applications, Elsevier.

[19] Lidl, R., \& Niederreiter, H. (1994). Introduction to Finite Fields and Their Applications, Cambridge University Press.

[20] McDaniel, W. L. (1996). Triangular numbers in the Pell sequence. The Fibonacci Quarterly, 34(2), 105-107.

[21] Shannon, A. G., Anderson, P. G., \& Horadam, A. F. (2006). Properties of Cordonnier, Perrin and Van der Laan numbers. International Journal of Mathematical Education in Science and Technology, 37(7), 825-831.

[22] Shannon, A. G., Horadam, A. F., \& Anderson, P. G. (2006). The auxiliary equation associated with the plastic number. Notes on Number Theory and Discrete Mathematics, 12(1), 1-12.

[23] Stakhov, A. P., \& Rozin, B. (2006). Theory of Binet formulas for Fibonacci and Lucas p-numbers. Chaos, Solitions \& Fractals, 27(5), 1162-1177.

[24] Stewart, I. (1996). Tales of a neglected number. Scientific American, 274(6), 102-103.

[25] Tasci, D., \& Firengiz, M. C. (2010). Incomplete Fibonacci and Lucas p-numbers. Mathematical and Computer Modelling, 52(9-10), 1763-1770. 